\title{
Numerical Modeling of Plasma - Flow Interaction
}

\author{
Jean-Charles Matéo-Vélez ${ }^{1}$, Francois Rogier ${ }^{1}$, \\ Frédéric Thivet ${ }^{1}$, and Pierre Degond ${ }^{2}$ \\ ${ }^{1}$ ONERA, Département Modèles pour l'Aérodynamique et l'Énergétique, \\ 2 avenue Edouard Belin, 31055 Toulouse, France \\ mateo@onera.fr \\ http://www.onera.fr \\ ${ }^{2}$ UMR 5640, Laboratoire Mathématiques pour l'Industrie et la Physique, \\ 118 route de Narbonne, 31062 Toulouse, France
}

\begin{abstract}
In the frame of the internal project PUMA (Plasma Used to Master Aerodynamics), ONERA is conducting fundamental studies of plasma-flow interactions. In this paper, the ionic wind created by corona discharges is studied in the case of a subsonic flow over a flat plate. The proposed mechanism of the ionic wind proposed is the addition of momentum by collisions between charged and neutral particles. In order to evaluate the effect of plasma on aerodynamics, a kinetic modeling of the discharge is coupled with a Fluid Dynamics code.
\end{abstract}

\section{Introduction}

A large number of studies has been carried out on the ionic wind since the 60's [1. This phenomenon was first discovered by Hauksbee in 1709. It is a way to provide energy to flows thanks to an electric power supply. The strong advantages of such an energy supply are that there is no need for external mass addition and it can act over a wide range of frequencies $(0-10 \mathrm{kHz})$. Numerous experiments [2]-6] have pointed out the possibility of adding significant momentum in the boundary layer of subsonic flows.

Boeuf and Pitchford [7] give a detailed description of the origin of ionic wind created by dielectric barrier discharges. When charged particles are submitted to an intense electric field $E$, they collide with the neutral molecules of the gas. By this way they can transfer an amount of their momentum. The force $f_{k \rightarrow n}^{i}$ that exerts a specie $k$ on a neutral $n$ in the direction $i$ is:

$$
f_{k \rightarrow n}^{i}=m_{k} N_{k} \nu_{k-n}\left(U_{k}^{i}-U_{n}^{i}\right)
$$

where $m_{k}$ is the mass of specie $k, N_{k}$ its density, $U_{k}^{i}$ the $i^{t h}$ component of its mean velocity vector; $\nu_{k-m}$ is the momentum transfer frequency for collisions between $k$ and $n$. This expression simplifies because the velocity of charged species are three or four orders of magnitude higher than the neutral velocity. 
Let us consider the density current $j_{k}^{i}$ and the mobility $\mu_{k}$ of specie $k$ :

$$
\begin{aligned}
j_{k}^{i} & =q N_{k} U_{k}^{i} \\
\mu_{k} & =\frac{q}{m_{k} \nu_{k-n}}
\end{aligned}
$$

where $q$ is the electric charge of specie $k$. Neglecting the gas velocity with respect to ions and electrons velocities, the volumetric force $f$ exerted by all the charged particles reads:

$$
f^{i}=\sum_{k} \frac{j_{k}^{i}}{\mu_{k}}
$$

It is important to note that all these contributions to the global force are algebraic. So, the total force can be equal to zero even if the single contributions are not equal to zero. The conductive density current $j_{c}^{i}$ is the sum of the particles current:

$$
\left|j_{c}^{i}\right|=\sum_{k}\left|j_{k}^{i}\right|
$$

And finally, the total density current $j_{t}^{i}$ is the sum of the conductive and displacement density currents and reads:

$$
\left|j_{t}^{i}\right|=\sum_{k}\left|j_{k}^{i}\right|+\varepsilon_{0} \frac{\partial E}{\partial t}
$$

where $\varepsilon_{0}$ is the dielectric permittivity of air.

Moreau's team [4] used a wire-to-wire discharge on a flat plate (Figure 1) and observed an ionic wind around $5 \mathrm{~m} / \mathrm{s}$ in the absence of uncoming flow. Two thin electrodes are flushed mounted in a dielectric plate. The first electrode is a 0.7 $\mathrm{mm}$ diameter anode set to a $+22 \mathrm{kV}$ potential. The second one has a $2 \mathrm{~mm}$ diameter and is set to $-10 \mathrm{kV}$. They are placed at a distance of $4 \mathrm{~cm}$. The regime of the discharge depends on many parameters such as the potential difference, the space between the electrodes, air humidity degree, flow velocity, etc. From the aerodynamic point of view, the most efficient regime is the corona regime in which a luminescent discharge appears in the vicinity of both electrodes. The purpose of this paper is to propose a model of this regime.

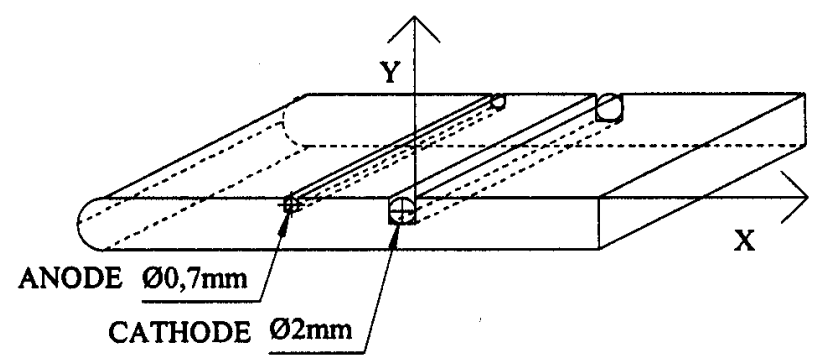

Fig. 1. Experimental setup of [4] 


\section{Modeling Corona Discharges - Flow Interaction}

A modeling of the experimental device proposed in [4] and described in section 1$]$ is proposed here. This modeling follows previous work [8].

\subsection{Space and Time Scales}

In order to simulate numerically the effect of corona discharges on a gas flow, two different time and space scales issues must be solved. First, to be correctly described, the discharge must be calculated with a maximal time increment of about $10^{-11} \mathrm{~s}$, which is representative of the kinetic time scale and the characteristic time of electron transport. In the same time, the characteristic response time of the flow to the discharge effect is about $10^{-3} \mathrm{~s}$. As a consequence, a full coupling of both parts of the problem is hardly realizable. The next three sections present the set of equations governing the problem, the numerical way of coupling and the results obtained.

\subsection{Equations}

The equations are written here in cartesian coordinates with the Einstein notation. The aerodynamic part of the problem follows the continuum and momentum equations (7) and (8):

$$
\begin{aligned}
\frac{\partial \rho U^{i}}{\partial x^{i}} & =0 \\
\rho\left(\frac{\partial U^{i}}{\partial t}+U^{j} \frac{\partial U^{i}}{\partial x^{j}}\right) & =-\frac{\partial P}{\partial x^{i}}+\mu \frac{\partial^{2} U^{i}}{\partial x^{j} \partial x^{i}}+f^{i}
\end{aligned}
$$

where $U^{i}$ is the $i^{\text {th }}$ component of the flow velocity vector, $\rho$ the volumetric mass of air, $P$ the pressure and $\mu$ the dynamic viscosity of air. The total electric force $f$ is calculated solving the density transport equations (9) for a set of $N$ particles species coupled with the Poisson equation (10) for the electric potential $V$ :

$$
\begin{aligned}
\frac{\partial N_{k}}{\partial t}+\frac{\partial N_{k} U_{k}^{i}}{\partial x^{i}} & =\omega_{k} \\
\Delta V & =-\frac{\rho_{c}}{\varepsilon_{0}} \\
\rho_{c} & =\sum_{k} Z_{k} N_{k}
\end{aligned}
$$

In these equations, $\omega_{k}$ is the rate of production of specie $k$. It is the balance between chemical production and decomposition. The $\Delta$ symbol stands for the divergence operator. $\rho_{c}$ is the space charge density, namely the difference between positive and negative charges densities. $Z_{k}$ is the charge of specie $k$.

The neutral particles velocity is the flow velocity $U^{i}$. The charged particles velocities are given by a drift-diffusion model. The ions velocity $U_{i o n}^{i}$ is the sum 
Table 1. Air plasma kinetics of 9

\begin{tabular}{|c|c|}
\hline Ionization & $\begin{array}{l}e, N_{2} \rightarrow e, e, N_{2}^{+} \\
e, O_{2} \rightarrow e, e, O_{2}^{+}\end{array}$ \\
\hline Attachment & $e, \mathrm{O}_{2} \rightarrow \mathrm{O}_{2}^{-}$ \\
\hline Recombination & $\begin{array}{l}e, N_{2}^{+} \rightarrow N_{2} \\
e, O_{2}^{+} \rightarrow O_{2} \\
O_{2}^{-}, O_{2}^{+} \rightarrow O_{2}, O_{2} \\
O_{2}^{-}, N_{2}^{+} \rightarrow O_{2}, N_{2}\end{array}$ \\
\hline Excitation & $e, O_{2} \rightarrow O_{2}^{*}$ \\
\hline Detachment & $O_{2}^{-}, O_{2}^{*} \rightarrow e, O_{2}, O_{2}$ \\
\hline Quenching & $O_{2}, O_{2}^{*} \rightarrow O_{2}, O_{2}$ \\
\hline
\end{tabular}

of the flow velocity and the drift velocity due to the electric field. The electrons velocity reads the same way, adding the diffusion velocity due to heterogeneity of their densities. Ions are heavy particles in comparison to electrons. As a consequence, their diffusion velocity is neglected :

$$
\begin{aligned}
U_{i o n}^{i} & =U^{i} \pm \mu_{i o n} E^{i} \\
U_{e}^{i} & =U^{i}-\mu_{e} E^{i}-\frac{D_{e}}{N_{e}} \frac{\partial N_{e}}{\partial x^{i}}
\end{aligned}
$$

Following 9, the discharge is described by the kinetics of air in high electric field which includes electrons, positive ions $\mathrm{O}_{2}^{+}$and $\mathrm{N}_{2}^{+}$, negative ions $\mathrm{O}_{2}^{-}$and metastables $O_{2}^{*}$. Table 1 gives the reactions taken into account in the present work. The kinetics coefficients corresponding to these reactions are given by [9] and $[10$.

The cathode is considered as a source of electrons thanks to ionic bombardment. The number of electrons $N_{e}^{b b t}$ emitted by this way is supposed proportional to the number of positive ions colliding with the cathode.

$$
N_{e}^{b b t}=\gamma \frac{N_{+}\left|U_{+}\right|}{\left|U_{e}\right|}
$$

where $\gamma$ is the ionic bombardment coefficient.

Finally, a resistance $R$ is connected in series between the anode and the power supply which voltage is $V_{0}$. The cathode is directly connected to the power supply mass. Thanks to eq. 6, the potential difference $\Delta V$ applied between the electrodes is:

$$
\Delta V=V_{0}-R I
$$

where $I$ is the total electric current in amperes. Its expression is given in the next section.

\subsection{D Discharge Model with Plasma Thickness}

Figure 2 presents the way the two parts of the problem (eq. (7)-(8) and eq. (9)-(14) ) will be coupled. The aerodynamic part is solved thanks to a Fluid 


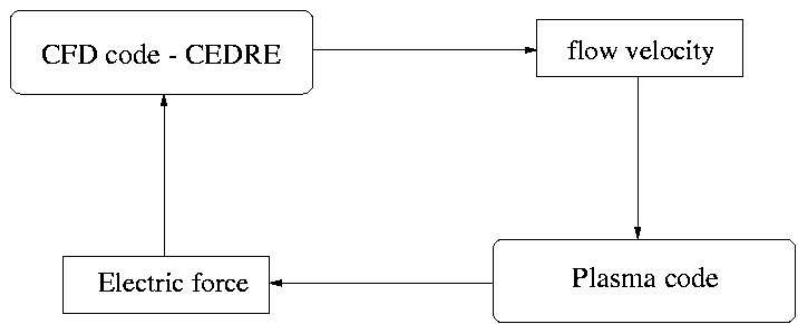

Fig. 2. Plasma - Aerodynamics Coupling

Dynamics code developed at ONERA, namely CEDRE. The space integration scheme follows a generalized MUSCL approach and the time integration scheme can be explicit ot implicit [12. For the flow calculation, the volumetric force exerted by the discharge is needed. The input data for plasma calculation is the global flow velocity. In this work, the discharge development in one space dimension without any effect of the external flow is first calculated. It consists in writing equations (9)-(14) in $1 \mathrm{D}$ and assuming $U=0$. This system is discretized by a finite volume method. The integration scheme is a second order scheme in time and space with a minmod slope limitor for the fluxes computation. The electric field $E_{s p}$ created by space charges is obtained thanks to a LU decomposition. The electric field $E_{\text {ext }}$ created by the electrodes with no space charge is calculated thanks to a $2 \mathrm{D}$ analytical formula [11, which permits to take into account the singularity of the two thin electrodes.

We suppose that all the variables (densities, velocities, electric potential, etc.) are constant over a thickness $S(x)$. It allows the total electric current conservation by ensuring the consistancy of the $2 \mathrm{D}$ electric field $E_{\text {ext }}$ with a $1 \mathrm{D}$ approximation. As a consequence, the equations (9)-(14) become:

$$
\begin{gathered}
\frac{\partial N_{k}}{\partial t}+\frac{1}{S} \frac{\partial S N_{k} U_{k}}{\partial x}=\omega_{k} \\
\frac{1}{S} \frac{\partial}{\partial x}\left(S \frac{\partial V}{\partial x}\right)=-\frac{\rho_{c}}{\varepsilon_{0}} \\
\rho_{c}=\sum_{k} Z_{k} N_{k} \\
U_{i o n}=U \pm \mu_{i o n} E \\
U_{e}=U-\mu_{e} E-\frac{D_{e}}{S N_{e}} \frac{\partial S N_{e}}{\partial x}
\end{gathered}
$$

According to (17), $S$ is obtained by solving $S(x) E_{\text {ext }}(x)$ equal to a constant. The intoduction of $S$ allows to consider a non trivial electric field respecting a zero divergence in 1D. The total current $I$ is the same in every cell of the meshing and is calculated with respect to eq. 21, where $L$ is the electrodes length:

$$
I=j_{t} L S
$$




\section{Results}

Here are presented the results obtained for the discharge development without any effect of an external flow. The physical and numerical parameters used for this simulation are presented in Table 2, where $N_{\text {mesh }}$ is the number of cells of the uniform mesh and $S_{\max }$ the maximal value of $S$. Figure 3 represents the evolution of the electric current. After an onset pulse followed by a long transient regime, the regular pulse regime plotted in Figure 3 is obtained. The frequency of these pulses is about $100 \mathrm{kHz}$. This description seems qualitatively close to the experimental results of [4] obtained for the same configuration (Figure 4). The minimal computed and experimental currents are about $0.8 \mathrm{~mA}$ and $0.5 \mathrm{~mA}$ respectively. Yet, the maximal computed current is one order of magnitude higher than the experimental one. This can be explained by two facts. First, the experimental potential difference is about $30 \mathrm{kV}$ instead of $40 \mathrm{kV}$ here. Then, the value of $S$ might be over estimated. This represents a limit of the 1D approach. 2D simulations would determine the thickness $S$ more accurately.

The fact that the pulsed discharge does not numerically appear for potential smaller than 35 or $40 \mathrm{kV}$ indicates that the kinetics model is under evaluated. We can argue for the absence of an additional electrons provider: the photoionization. Yet, this regime seems to be similar to the Trichel pulses regime of negative corona discharges. So the simulation of the cathode region is quite in good agreement with previous works.

Figure 5 represents the evolution of electron density during a single pulse. On Figures 5, 6] and 7, the time $t=0 \mathrm{~s}$ corresponds to the ignition of a single pulse. The pulses observed in Figure 3 are due to the periodic creation of electrons thanks to ionization. When the space charge is high enough to diminish the electric field at the electrodes (Figure 6), the discharge is stopped and the species are evacuated. When the electric field reaches its initial value, a new pulse initiates.

Table 2. Parameters

\begin{tabular}{|l|l|l|l|l|}
\hline$V_{0}$ & $R$ & $\gamma$ & $N_{\text {mesh }}$ & $S_{\max }$ \\
\hline $40 \mathrm{kV}$ & $10 \mathrm{k} \Omega$ & $10^{-4}$ & 400 & $5 \mathrm{~mm}$ \\
\hline
\end{tabular}

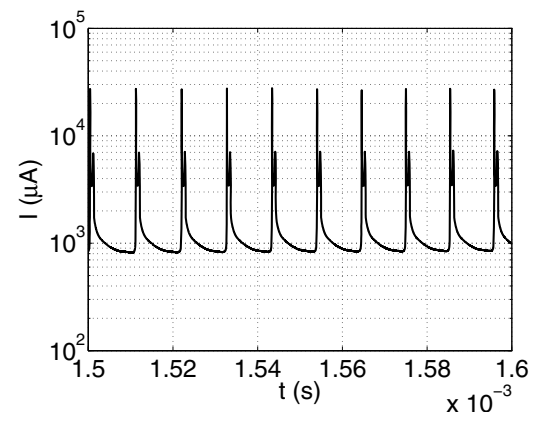

Fig. 3. Calculated electric current

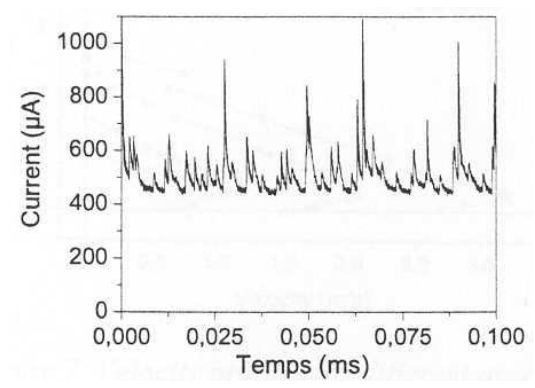

Fig. 4. Exp. electric current 4 ] 


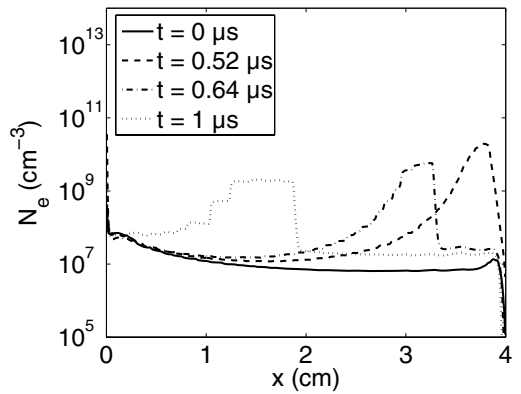

Fig. 5. Repartition of electron number density. $t=0 \mathrm{~s}$ corresponds to the beginning of a pulse.

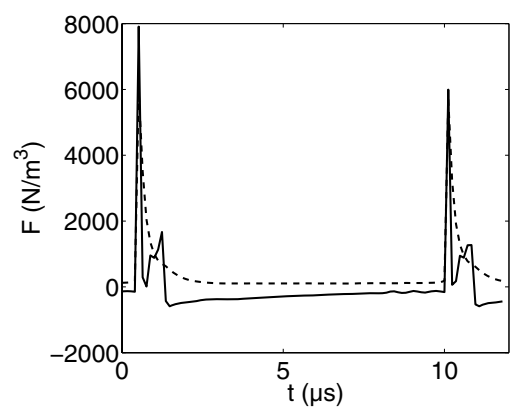

Fig. 7. Force at the anode $(-)$ and at the cathode (- -)

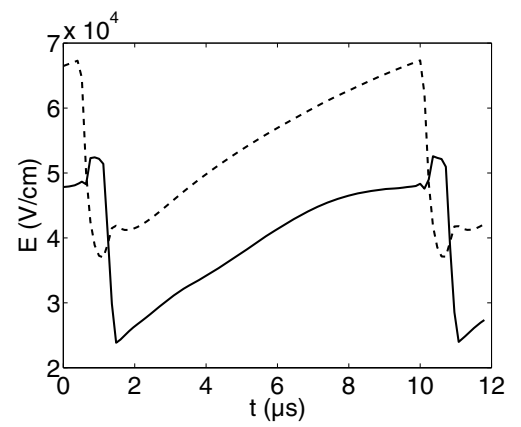

Fig. 6. Evolution of the electric field at the anode (-) and at the cathode (- -)

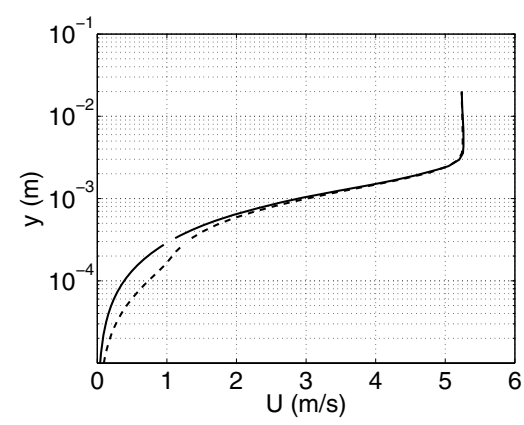

Fig. 8. flow velocity profile without plasma force $(-)$ and with plasma force $(--)$

From the aerodynamic point of view, the forces exerted by the discharges are also periodic. One of the most prominent features is that the forces are concentrated in the cathode and anode regions. The force levels are very high there, about 1000 to $5000 \mathrm{~N} / \mathrm{m}^{3}$. There is almost no force in the space between the electrodes because the density currents of positive and negative ions $j_{+}$and $j_{-}$are almost equal, but with opposite signs, see eq. (4). Figure 7 shows the evolution of the force near the anode and the cathode. The forces are locally important near electrodes but weak on average. The CFD code CEDRE has been used in order to simulate the effect of the calculated forces on a subsonic laminar flow over a flat plate. The external flow velocity is $5 \mathrm{~m} / \mathrm{s}$. Figure 8 presents the flow velocity profiles obtained at location $X=1 \mathrm{~mm}$ on Figure 1 when applying or not applying the force. The gain of velocity is obtained near the electrodes and is about $0.5 \mathrm{~m} / \mathrm{s}$. This influence is smaller than observed in experiments 4, where the gain of velocity can reach $1 \mathrm{~m} / \mathrm{s}$ on a thickness of $5 \mathrm{~mm}$, for an uncoming flow of $5 \mathrm{~m} / \mathrm{s}$. The force is insufficient to explain the experimental velocity gains because it is located just around both electrodes. To act more efficently on flows, this force should apply on a larger region. 


\section{Conclusion}

In order to represent the creation of ionic wind by corona discharges, this paper proposes a simple coupling between a plasma kinetics model and a flow model. The results of the plasma simulations show a good agreement with the Trichel pulses. Once applied to the gas flow, the electric force obtained by the plasma simulations seems insufficient to explain the ionic wind experimentally observed 4]. Consequently, the efforts are now focused on enriching the kinetics of the positive corona discharges by taking into account the photoionization process, as well as plasma-flow coupling.

\section{Aknowledgements}

The authors would like to thank G. Quinio from ONERA who realized the first version of the code developed in this work [13, and J. P. Boeuf from CPAT for a number of usefull discussions. The first author thanks DGA for supporting his research.

\section{References}

1. Robinson M.: Movement of air in the electric wind of the corona discharge. AIEE transactions, New York, USA (1961).

2. Shcherbakov Y. V. and coll.: Drag reduction by AC streamer corona discharges along a wing-like profile plate. $31^{\text {st }}$ AIAA Plasmadynamics and Lasers Conference, Denver, USA, AIAA paper 2000-2670 (2000).

3. Roth J. R., Sin H., Madhan R., Wilkinson S.: Flow re-attachment and acceleration by paraelectric and peristaltic electrohydrodynamic effects. $41^{\text {st }}$ Aerospace Sciences Meeting and Exhibit, Reno, USA, AIAA paper 2003-531 (2003).

4. Pons J., Moreau E., Touchard G.: Surface DC corona discharges and AC barrier discharges in ambient air at atmospheric pressure: measurements of the induced ionic wind velocity. $15^{\text {th }}$ International Conference on Gas Discharges and their Applications, Toulouse, France (2004).

5. Post M. L., Corke T. C.: Plasma actuators for separation control on airfoils. $11^{\text {th }}$ International Symposium on Flow Visualization, Notre-Dame, USA (2004).

6. Zavialov I. N., Roupassov D. V., Starikovskii A. Yu., Saddoughi S. G.: Boundary layer control by gas discharge plasma. European Conference for Aerospace Sciences (EUCASS), Moscow, Russia (2005).

7. Boeuf J. P., Pitchford L. C.: Electrohydrodynamic force and aerodynamic flow acceleration in surface barrier discharge. J. Appl. Phys. 97, 103307 (2005).

8. Mateo-Velez J. C., Thivet F., Rogier F., Degond P., Quinio G.: Numerical modeling of corona discharges and their interaction with aerodynamics. European Conference for Aerospace Sciences (EUCASS), Moscow, Russia (2005).

9. Morrow R.: The theory of positive glow corona. J. Phys. D: Appl. Phys. 30, 30993114 (1997).

10. Bolsig, CPAT \& Kinema Software, http://www.siglo-kinema.com/bolsig.htm.

11. Li S. Z., Uhm H. S.: Investigation of electrical breakdown characteristics in the electrodes of cylindrical geometry. Phys. Plasmas 11, 3088-3095 (2004). 
12. Chevalier P., Courbet B., Dutoya D., Klotz P., Ruiz E., Troyes J., Villedieu P.: CEDRE, Development and Validation of a Multiphysic Computational Software. European Conference for Aerospace Sciences (EUCASS), Moscow, Russia (2005).

13. Quinio G.: Modélisation numérique de la génération d'un plasma d'air dans un écoulement. PhD thesis, INSA Toulouse, France (december 2005). 